\title{
Survey of colour contrast sensitivity in non-ophthalmic users of blue-green wavelength argon lasers
}

\author{
Louise E Allen, Andrew J Luff, Christopher R Canning
}

\begin{abstract}
Background-Previous studies have shown that ophthalmologists using bluegreen argon laser may suffer subtle defects in their colour vision. A reduction in colour contrast sensitivity in the tritan colour confusion axis, an early manifestation of blue cone photoreceptor injury by the high energy photons of the laser, has been demonstrated and has prompted a reappraisal of laser safety in ophthalmology. Argon laser is also frequently used in scientific research, often at higher power output and for longer periods than is used in clinical practice. The scientists operating these lasers are at risk of developing similar phototoxic retinal injury.

Methods-The colour contrast sensitivity of 18 scientists who regularly use short wavelength argon laser was investigated. Results-Eye protection was infrequently used and individuals had been subjected to between 580 and 7200 hours of cumulative laser exposure during the course of their research.

Conclusion-The use of blue-green argon laser by the scientists investigated was not associated with a significant reduction in colour contrast sensitivity.

(Br F Ophthalmol 1995; 79: 332-334)
\end{abstract}

Prolonged exposure to short wavelength light is known to cause phototoxic injury to the retina, the blue cone photoreceptors being especially susceptible. ${ }^{12}$ In natural circumstances, sufficient exposure to cause photoreceptor damage does not occur, but the use of short wavelength laser in clinical, industrial, and scientific settings may pose risks to the operator unless adequate eye protection is employed.

Psychophysical measurements, such as the Hardy Rittler Rand, Farnsworth Munsell 100 hue tests and City University plates, have traditionally been used to test colour discrimination clinically. However, a new test of colour contrast sensitivity using computer graphics has proved to be a much more sensitive and reproducible technique. ${ }^{34}$ Using this technology, Arden et al have shown a subtle reduction of colour contrast sensitivity in the tritan colour confusion axis in ophthalmologists using blue-green (wavelength $488 \mathrm{~nm}$ ) argon laser for retinal photocoagulation as a result of unprotected flashbacks from the contact lens surface. 356
Scientists are also exposed to argon laser light and manual alignment of the unshielded laser beam between mirrored surfaces is often an integral part of their experiments. The aim of this study was to determine whether retinal phototoxicity was occurring in non-ophthalmic users of blue-green argon laser and to emphasise the importance of eye protection in this population.

\section{Method and materials}

SUBJECTS

An invitation for colour contrast sensitivity testing was sent to the laser users in the physics, chemistry, physiology, and oceanography departments at Southampton University. Of those scientists screened, the 19 who regularly used blue-green argon laser in their research were considered suitable subjects for inclusion in the study. Each subject completed a questionnaire regarding relevant ophthalmic and medical history, laser use, and the eye protection utilised. All initially underwent visual acuity testing and gross colour vision screening with City University plates.

COLOUR CONTRAST SENSITIVITY ASSESSMENT Colour contrast sensitivity assessment using a high quality colour monitor displaying graphics controlled by a personal computer was first described by Arden et al in 1988.4 In this study the colour contrast threshold of the right eye for each colour axis was recorded by the same observer with the subjects sitting 1.5 metres away from the monitor in a darkened room.

Initially, the subject's spectral sensitivity was measured using heterochromatic flicker photometry. These data allow subsequent colour contrast threshold testing, using computer generated graphics of isoluminant hues, along personalised protan, deutan, and tritan colour confusion axes. Colour contrast sensitivity was assessed by a modified binary search scheme; the colour contrast between the central, flashing optotype and the constant background being adjusted automatically in a mathematical progression until the letter was perceived. Colour contrast was defined as $0 \%$ when the optotype and the background were the same hue and $100 \%$ when the difference between them was the maximum achievable by the colour monitor. The colour contrast threshold was the minimum percentage colour contrast required for the identification of the letter. \\ L E Allen \\ A J Luff \\ 16 Nover
}



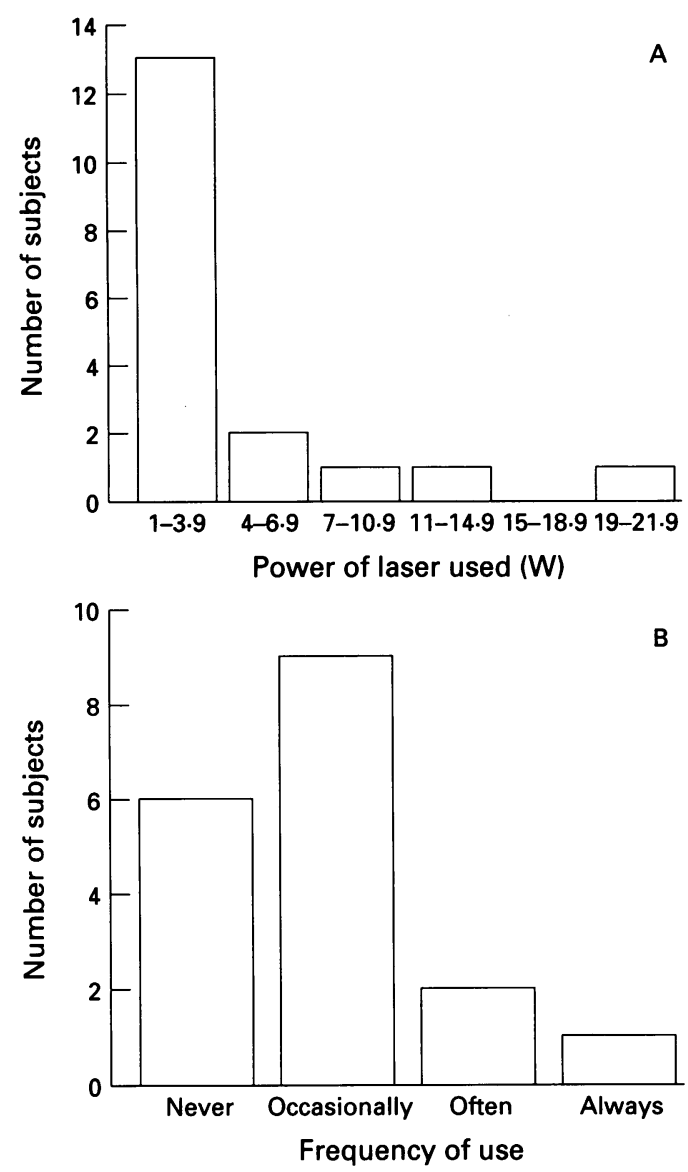

Figure 1 (A) Power of laser used. (B) Use of protective goggles.

Previous measurements of colour contrast sensitivity in healthy volunteers have demonstrated a mean colour contrast threshold of $4.5 \%$ for all three axes, the upper limit of normal being $7 \cdot 7 \%(2.5 \mathrm{SD}$ from the mean $) .^{5}$

\section{Results}

All subjects tested were aged between 22 and 52 years and had used argon laser for more than 1 year, with a mean weekly exposure of 15 hours. None had used a laser within 24 hours of testing. The laser systems utilised in their studies were open (that is, no shielding

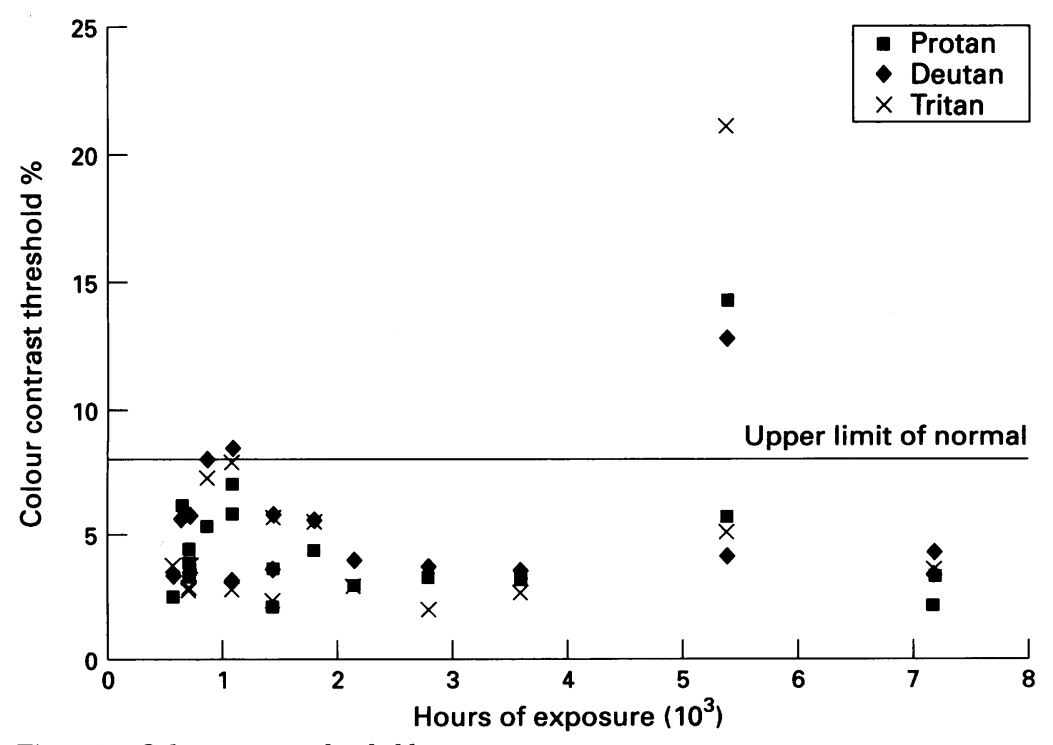

Figure 2 Colour contrast thresholds. between the laser beam and the observer) and the power used ranged from 1 to $20 \mathrm{~W}$ (Fig 1A). Furthermore, initial manual alignment of the laser beam was necessary, precluding the regular use of protective goggles which would render the beam invisible (Fig 1B).

The subjects had corrected visual acuities of $6 / 6$ or better. One subject showed protanomaly on testing with City University colour plates and was excluded. One scientist was an insulin dependent diabetic of 10 years' duration. $\mathrm{He}$ had no previous ophthalmic history and was included in the study.

The colour contrast thresholds were plotted with respect to cumulative laser exposure (Fig 2) and analysed statistically. The two patients who had used laser the longest (over 7000 hours' exposure) were also the oldest studied at 44 and 52 years of age. Two subjects had raised colour contrast thresholds: a slightly raised deutan threshold was detected in a 26-year-old physicist who had used the laser for approximately 1080 hours at a power of $6 \mathrm{~W}$. The other, with raised colour contrast thresholds in all colour axes, was a 33-year-old diabetic who had used the laser for approximately 5400 hours at a power of $1 \mathrm{~W}$.

\section{STATISTICAL ANALYSIS}

The data are summarised in Table 1. Comparison between the colour contrast sensitivity in the tritan axis and that in the protan and deutan axes was made using a Wilcoxon sum rank test (Table 2). A Spearman rank correlation test analysed the relation between the colour contrast thresholds and cumulative hours of laser exposure (Table 3). There was no significant difference in contrast threshold between protan and tritan axes $(p=0.695)$ and the correlation of tritan contrast threshold with cumulative laser exposure was also insignificant (correlation coefficient $=0 \cdot 047$ ).

\section{Discussion}

Several case controlled studies have identified ophthalmologists who use blue-green argon laser for retinal photocoagulation as being at risk of phototoxic injury to the blue cones of the macula. It has been suggested that the reflections, or flashbacks, of the low power argon aiming beam off the lens surface are responsible for this. Initially this temporarily impairs tritan colour contrast sensitivity but cumulative exposure can result in a permanent colour vision defect. ${ }^{3}$ Long term exposure to blue light has been shown to lead to histological changes of photoreceptor damage in monkey retinas. ${ }^{1}$ Most argon laser systems now incorporate short wavelength filters in the eyepieces which protect the operator from flashbacks and more recent laser systems utilise a helium-neon laser aiming beam.

There are numerous scientific uses for lasers, many requiring an open laser system where the operator is directly exposed to the laser beam and its reflection from the mirrored aligning surfaces (Fig 3). The scientists operating blue-green argon laser might be expected 
Table 1 Statistical analysis of colour contrast thresholds and cumulative hours of laser exposure

\begin{tabular}{llcccc}
\hline & Mean & Median & $\begin{array}{l}\text { Standard } \\
\text { deviation }\end{array}$ & $\begin{array}{l}\text { First } \\
\text { quartile }\end{array}$ & $\begin{array}{l}\text { Third } \\
\text { quartile }\end{array}$ \\
\hline Exposure (hours) & 2492 & 1440 & 2282 & 720 & 4050 \\
Protan axis & $4 \cdot 628$ & $3 \cdot 704$ & $2 \cdot 800$ & $3 \cdot 142$ & $5 \cdot 771$ \\
Deutan axis & $5 \cdot 124$ & $4 \cdot 036$ & $2 \cdot 467$ & $3 \cdot 516$ & $5 \cdot 771$ \\
Tritan axis & $4 \cdot 920$ & $3 \cdot 49$ & $\mathbf{4} \cdot 33$ & $2 \cdot 79$ & $5 \cdot 56$ \\
\hline
\end{tabular}

Table 2 Comparison of colour contrast thresholds between colour confusion axes (Wilcoxon rank sum test)

\begin{tabular}{lcc}
\hline & Wilcoxon statistic & p Value \\
\hline Protan axis-deutan axis & 53.0 & 0.163 \\
Protan axis-tritan axis & 76.0 & 0.695 \\
Deutan axis-tritan axis & 130.0 & 0.055 \\
\hline
\end{tabular}

Table 3 Correlation between cumulative hours of laser exposure and colour contrast sensitivity (Spearman rank test)

\begin{tabular}{lr}
\hline & Correlation coefficient \\
\hline Protan threshold and hours of exposure & -0.141 \\
Deutan threshold and hours of exposure & 0.088 \\
Tritan threshold and hours of exposure & 0.047 \\
\hline
\end{tabular}

to demonstrate reduced tritan contrast sensitivity, similar to that documented in ophthalmologists, resulting from cumulative blue cone receptor injury.

Colour contrast thresholds do not correlate significantly with age, ${ }^{6}$ therefore an upper limit of normal of $7 \cdot 7 \%$ was used for all subjects in this study. We have assumed that the test results from the right eye were representative since all subjects viewed the open laser beam binocularly. The diabetic subject, who had no clinical evidence of retinopathy, was the only subject with a marked elevation in tritan, deutan, and protan thresholds; poor colour discrimination in diabetics with little or no retinopathy has been previously recognised. ${ }^{7} 8$

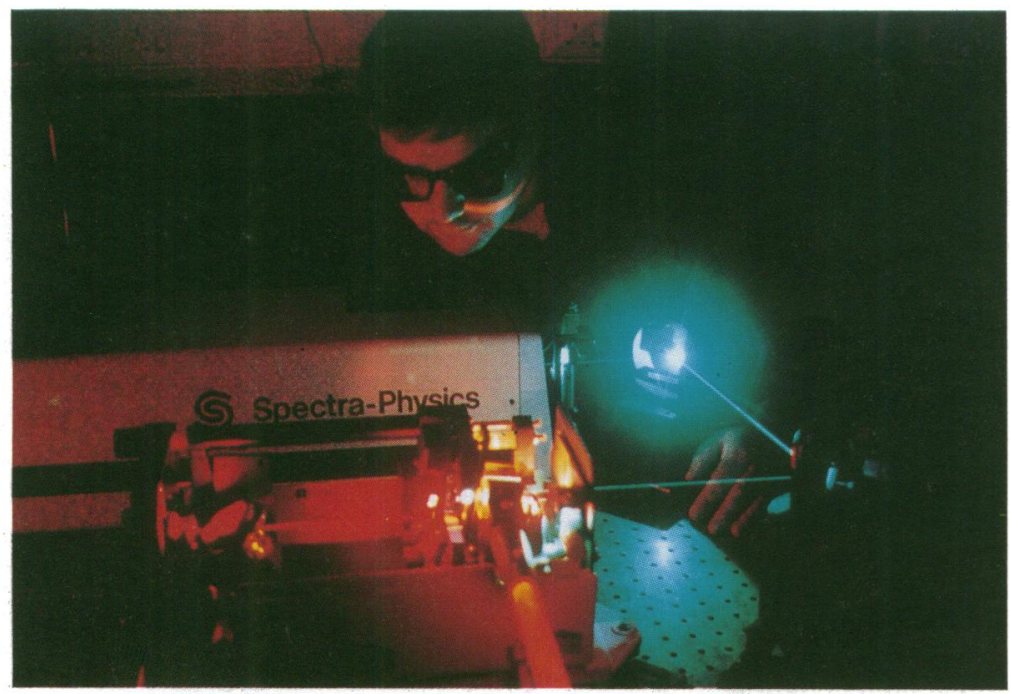

Figure 3 Argon and helium-neon laser in use.
Another subject, who had used laser for approximately 1080 hours, had a slightly raised deutan threshold of questionable significance.

The scientists studied used higher power laser for longer periods than ophthalmologists and were exposed to unshielded reflections during laser alignment similar to those implicated in the pathogenesis of retinal injury in ophthalmologists. Although one might have predicted a similar colour vision disturbance to that identified in ophthalmologists, this study neither demonstrated the expected reduction in tritan contrast sensitivity nor a correlation between laser exposure and tritan contrast sensitivity in this group of scientists. The absence of evidence for cumulative blue cone damage is puzzling. A possible explanation is that while ophthalmologists receive foveal flashbacks from the contact lens throughout retinal photocoagulation, the aligning process in scientific lasers takes a fraction of the experiment's duration. The scientists studied may have actually received fewer harmful flashbacks than the ophthalmologists previously studied, even though the overall exposure time was longer.

Short term defects in colour contrast thresholds following periods of argon laser exposure in ophthalmologists are known to predate permanent elevation. ${ }^{5}$ Since this study was carried out 24 hours after laser use, it does not exclude the presence of temporary colour contrast defects occurring directly after laser operation. We plan further studies to determine the presence of such short term defects in this population.

Improvements in the design of scientific laser systems, protective eye wear, and publicity of the colour vision disturbance affecting clinical laser users may improve the scientific community's awareness and compliance with eye protection, thereby further reducing the risk of phototoxic retinal injury.

We thank Professor T Shelley and Mr H E Blayden for thei advice and assistance.

1 Ham WT, Harold A, Mueller JJR, DuPont G III, Guerry RK. Action spectrum for retinal injury from near-violet radiation in the aphakic monkey. Am f Ophthalmol 1982 93: 229-306

2 Hawerth RS, Sperling HG. Prolonged color blindness induced by intense spectral lights in the Rhesus monkey. Science 1971; 114: 520-3

3 Gunduz K, Arden GB. Changes in colour contrast sensitivity associated with operating argon lasers. $\mathrm{Br} \mathcal{F}$ Ophthalmo 1989; 73: 241-6.

4 Arden GB, Gundez K, Perry S. Colour vision testing with a computer graphics system. Clin Vis Sci 1988; 12: 303-21.

5 Berninger TA, Canning CR, Gunduz K, Strong N, Arden GB. Using argon laser blue light reduces ophthalmologists' color contrast sensitivity. Arch Ophthalmol 1989; 107 1453-8.

6 Arden GB, Berninger TA, Hogg CR, Perry S. A survey of colour discrimination in German ophthalmologists. Ophthalmology 1991; 98: 567-75.

7 Roy MS, Gunkel RD, Podgor MJ. Color vision defects in early diabetic retinopathy. Arch Ophthalmol 1986; 104: 225-8.

8 Bresnick GH, Condit RS, Palta M, Korth K, Groo A, Syrjala $S$. Association of hue discrimination and diabetic retinopathy. Arch Ophthalmol 1985; 103: 1317-24. 